\title{
The directivity of solar type III bursts at hectometer and kilometer wavelengths: Wind-Ulysses observations
}

\begin{abstract}
X. Bonnin, S. Hoang, and M. Maksimovic
Observatoire de Paris, LESIA, UMR 8109 CNRS, 92195 Meudon, France

e-mail: xavier . bonnin@obspm. fr

Received 13 March 2008 / Accepted 28 May 2008

ABSTRACT

We investigate the directivity of solar type III radio bursts at hectometer and kilometer wavelengths using radio data recorded simultaneously by the Wind and Ulysses spacecraft which are widely separated in heliocentric longitude and latitude. From the positions of the associated flares and the solar wind speed and plasma density measured aboard the spacecraft, we estimate the location of the type III radio sources along the approximately spiral magnetic field lines connected to the flare sites. Using the ratios of the radio flux densities measured from 1995 to 2005 at the same frequencies $(940-80 \mathrm{kHz})$ on these spacecraft, we have for the first time derive the average directivity diagram of type III bursts in two dimensions, longitude and latitude relative to the emission source. This diagram is found to exhibit no significant variation with the solar activity. The diagram in longitude is found to shift east of the local magnetic field direction at the source. The angular width and eastward shift of the diagram are observed to increase with decreasing frequencies; around $800 \mathrm{kHz}$, its full width at maximum $/ 10$ level is about $190^{\circ}$ and its eastward shift $23^{\circ}$. The diagram in latitude, obtained uniquely thanks to the out-of-ecliptic orbit of Ulysses, shows no significant variation with latitude.
\end{abstract}

Key words. Sun: radio radiation - Sun: particle emission - plasmas - methods: data analysis

\section{Introduction}

It is generally agreed that the solar type III radio bursts are generated by suprathermal electrons $(v \approx 0.03$ to $0.3 \mathrm{c})$, ejected from solar active regions, accelerated in the low corona, and traveling outward along open magnetic field lines into the interplanetary medium (IPM). Along their path, these electrons develop "bump-on-tail" distribution unstable to the production of Langmuir waves at the local plasma frequency $f_{\mathrm{p}}(\mathrm{kHz}) \simeq 9 n_{\mathrm{e}}^{1 / 2}$, where $n_{\mathrm{e}}$ is the plasma density in $\mathrm{cm}^{-3}$. Some of the Langmuir wave energy is then converted through nonlinear wave-wave interactions into electromagnetic waves at the fundamental and/or second harmonic of $f_{\mathrm{p}}$. Since the energetic electrons are guided along the open field lines, the "bump-on-tail" instability occurs essentially along these lines. As a result, the type III emission is expected to radiate primarily in about the magnetic field direction. On the other hand, the propagation of radio waves through the inhomogeneous IPM is controlled by large-scale structures (refraction and focusing) and small-scale inhomogeneities (random scattering) of the plasma density. These propagation effects are likely to significantly modify the primary directivity of the radio emission, resulting in a very different apparent directivity that is actually observed.

Knowledge of the emission directivity is of very important when studying the emission mechanism and ubiquitous propagation effects in the medium, particularly at low frequency. The first stereoscopic observations of the type III directivity were carried out at $169 \mathrm{kHz}$ by Caroubalos \& Steinberg (1974) and Caroubalos et al. (1974) from the Earth and Soviet planetary probe Mars-3. A thorough documentation of these works at high frequencies was made by Poquérusse (1984). At lower frequencies, namely at the hectometer and kilometer wavelengths, the widespread visibility of the type III emission was reported by MacDowall (1982), Dulk et al. (1985), and
Lecacheux et al. (1989), using longitudinally separated spacecraft in the ecliptic plane, ISEE-3 and Voyagers. The first stereoscopic directivity measurements were reported by Poquérusse et al. (1996) and Hoang et al. (1997), using comparisons of flux densities measured between the ARTEMIS ground-based radio spectrograph around $150 \mathrm{MHz}$ and the radio receiver (1.25$940 \mathrm{kHz}$ ) on the Ulysses spacecraft at the highest frequencies. Due to intensity intercomparisons performed between two very different frequency domains, these authors had to assume the radio spectrum to remain unchanged for all bursts, on the one hand, and, on the other, to select bursts occurring only near the SunEarth line for the ground-based spectrograph to always be set in the direction of the burst maximum intensity for comparison.

In this paper, we present the first stereoscopic measurement of the hectometer and kilometer type III directivity using radio data acquired simultaneously by the Wind and Ulysses spacecraft at the same frequencies. The advantage in combining them for stereoscopic radio observations is twofold: (1) Ulysses is widely separated from Wind in heliocentric longitude and latitude. (2) Both radio receivers are well-calibrated and can operate a common list of frequencies for accurate intercomparisons. The second requirement alleviates the difficulties mentioned above, encountered by Poquérusse et al. (1996) and Hoang et al. (1997) in their works based on intensity comparisons between two very different frequency domains. In Sect. 2 we describe the observations and data selection. In Sect. 3 we explain our method for measuring the directivity of the type II burst. Section 4 presents the results discussed in Sect. 5. In Sect. 6, we give a summary and conclusions.

\section{Observations and data selection}

We use radio data from the Unified Radio and Plasma Wave (URAP) experiment on Ulysses (Stone et al. 1992) and the 
WAVES instrument on Wind (Bougeret et al. 1995). The radio receiver on Wind (RAD1, 20-1040 kHz, $3 \mathrm{kHz}$ bandwidth) can be commanded to operate on nearly all the 12 frequency channels of the high-band receiver on Ulysses $(52-940 \mathrm{kHz}, 3 \mathrm{kHz}$ bandwidth): 940, 740, 540, 387, 272, 196, 148, 120, 100, 81, 63, and $52 \mathrm{kHz}$. Both these receivers are well-calibrated and have the 2-D direction-finding using the spacecraft spin modulation of the radio signal (Manning \& Fainberg 1980; Fainberg et al. 1985). For direction-finding, the antenna system used on Ulysses (spin period $\simeq 12 \mathrm{~s}$ ) consists of the spin-axis electric monopole of $7.5 \mathrm{~m}$ and spin-plane electric dipole of $2 \times 35 \mathrm{~m}$; on Wind ( spin period $\simeq 3 \mathrm{~s}$ ), it is composed of the spin-axis electric dipole of $2 \times 5.28 \mathrm{~m}$ and spin-plane electric dipole of $2 \times 50 \mathrm{~m}$.

In the present study, we have selected type III bursts observed simultaneously by Wind and Ulysses between 1995 and 2005, a period spanning the 1996 minimum and 2001 maximum of the solar cycle 23. During that time, Ulysses made two orbits over the Sun's poles and explored wide ranges of distance, latitude, and longitude in the heliocentric Earth ecliptic (HEEC) coordinate system. Wind moved along its distant in-ecliptic prograde orbits around Earth to successively explore the Earth's magnetosphere and solar wind. We have considered bursts observed only when Wind was located in the solar wind well upstream of the Earth's bow shock, when the bursts were not polluted by terrestrial kilometric radiation, were relatively simple, isolated, and intense enough (at least $4 \mathrm{~dB}$ above background) so that their intensity time profiles at each frequency could be measured reliably at both spacecraft. In all cases, the background was first substracted by using quiet periods between bursts. The importance of properly measuring the burst intensity time profiles will appear clearer in Sect. 3.2. Another criterion used in selecting type III bursts is their association with solar flares within NOAA active regions (AR). This association allows us to know, within about $25^{\circ}$, the position of bursts near the Sun, as further explained in Sect. 3.1. For that purpose, we searched for a solar $\mathrm{H} \alpha$ and/or X flare could be associated in time with each selected type III. The associated solar flare was identified within an AR visible on the Sun using the Solar Geophysical Database (http://sgd.ngdc.noaa.gov/sgd/jsp/solarindex.jsp). Whenever an ambiguity existed on the burst position near the Sun, we checked it by using the direction-finding on the Wind radio receiver, and when available, meter wave type III bursts recorded by the RadioHeliograph at Nançay (France) (http://secchirh.obspm.fr).

Finally, from about 2000 bursts initially selected for the study, we are left with about 1000 bursts could be unambiguously associated with an identified AR. This type III data set does serve as a basis for measuring the radiation diagram as will be described below.

\section{Measuring the type III radiation diagram}

In this section we derive the 2-D directivity diagram of the hectometric and kilometric type III using the burst data set described above.

\subsection{Source location and observing reference frame}

To derive the radiation diagram, we need to know the 3-D position in the IPM of the type III source from which we can define the different viewing directions for Ulysses and Wind. To determine the position, we follow the method described by Saito et al. (1977) and Leblanc et al. (1998). We assume that the solar flare electrons responsible for the type III follow the approximately Archimedean spiral open magnetic field lines rooted in the Sun near the center of the associated ARs (see Sect. 2) and spreading outward into the IPM at the same constant heliolatitudes as the ARs. In the HEEC coordinates the spiral field lines can be expressed as

$r-r_{\mathrm{AR}}=-\frac{v_{\mathrm{SW}}}{\omega_{\odot}}\left(l-l_{\mathrm{AR}}\right)$

where the subscript AR denotes the related active region, $r$ is the heliodistance, $r_{\mathrm{AR}}$ the reference distance of the spiral field line rooted in the AR (assumed at 1 solar radius), $l$ the heliolongitude, $l_{\mathrm{AR}}$ the longitude of the footpoint of the spiral (assumed to be the AR's center), $v_{\text {sw }}$ the solar wind speed, and $\omega_{\odot}$ the solar corotational angular speed of $13.3^{\circ}$ per day.

We can estimate the value of $v_{\text {sw }}$ using solar wind speed recorded in situ at Wind (Ogilvie et al. 1995) located near Earth. If we assume that these spirals remain unchanged, i.e., with negligible radial and temporal evolution, then the value of $v_{\mathrm{sw}}$ is measured by Wind at the time $t_{\mathrm{W}}$ when the spiral line rooted in the associated AR passes the spacecraft by corotation.

Similarly, we can represent the plasma conditions all along the spiral paths travelled by the type III electrons, using the plasma density measured in situ at Wind (Ogilvie et al. 1995) at the time $t_{\mathrm{W}}$ and the fact that in the IPM the density decreases closely as $r^{-2}$ (McComas et al. 1992; Issautier et al. 1998). This procedure allows complete determination of the position of the type III sources along the spiral field lines at different frequencies.

Figure 1 illustrates the geometry of observation. Figure 1 (left panel) shows the type III source along the spiral magnetic field line and the locations of Wind and Ulysses in the HEEC coordinate system $x y z$. Figure 1 (right panel) defines the new observation reference frame centered at the type III source: the $x^{\prime}$-axis is tangent to the open magnetic field line passing the source antisunward; the $z^{\prime}$-axis is in the plane defined by the $x^{\prime}$-axis and the vertical to the ecliptic plane, oriented in the direction of ecliptic North; the $y$ '-ais completes the orthogonal right handed coordinate system. In the source-centered reference frame just defined, the new coordinates of the observing spacecraft (with index $i=U$ for Ulysses, $W$ for Wind) are: the spacecraft Cartesian components $x_{i}^{\prime}, y_{i}^{\prime}, z_{i}^{\prime}$, which are transformed from the spacecraft HEEC Cartesian coordinates $x_{i}, y_{i}, z_{i}$; and the coordinates of the spacecraft relative to the source frame, namely its longitude $\varphi_{i}=\arctan \left(y_{i}^{\prime} / x_{i}^{\prime}\right)$ with $\varphi_{i} \in\left(-180^{\circ} \mathrm{E},+180^{\circ} \mathrm{W}\right)$, its latitude $\theta_{i}=\arctan \left(\sqrt{x_{i}^{\prime 2}+y_{i}^{\prime 2}} / z_{i}^{\prime}\right)$ with $\theta_{i} \in\left(-90^{\circ} \mathrm{S},+90^{\circ} \mathrm{N}\right)$, and its distance to the source $d_{i}=\sqrt{{x_{i}^{\prime 2}}^{2}+y_{i}^{\prime 2}+z_{i}^{\prime 2}}$.

\subsection{Radiation diagram}

At the antenna terminals of each spacecraft with index $i$, we measure the source flux density time profile, $S_{i}(t)\left(\mathrm{Wm}^{-2} \mathrm{~Hz}^{-1}\right)$. Figure 2 shows an example of a type III burst observed by Wind (top) and Ulysses (bottom) on 17 June 1996 at a frequency of $196 \mathrm{kHz}$. The continuous curve above the background level represents the actual burst flux density profile. The lower curve, denoted above as $S_{i}(t)$, shows the same data, but with the background removed. The burst onset time, $t_{\text {onset }}$, defines the time at which the burst intensity rises significantly from background. The end time, $t_{\mathrm{end}}$, is the time when the burst intensity goes down to the background level. 

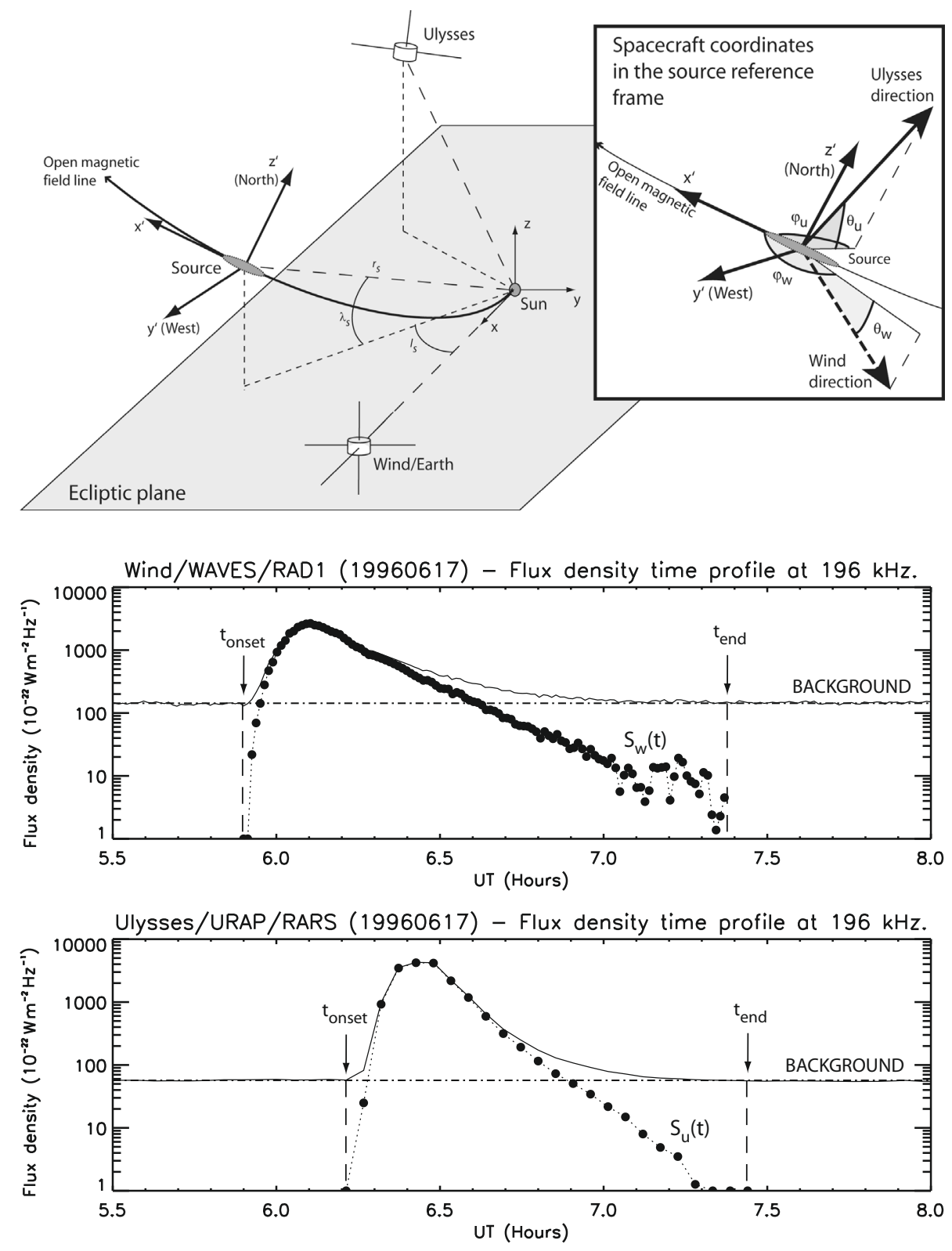

Fig. 1. Geometry of observation. (Left panel) Type III source on the spiral open magnetic field line and the locations of Wind and Ulysses in the HEEC (Heliocentric Earth Ecliptic) coordinate system xyz. (Right panel) Sourcecentered reference frame $x^{\prime} y^{\prime} z^{\prime}$ : the $x^{\prime}$-axis is tangent to the open magnetic field line passing the source anti-sunward; the $z^{\prime}$-axis is in the plane defined by the $x^{\prime}$-axis and the vertical to the ecliptic plane, oriented to ecliptic North; the $y^{\prime}$-axis completes the orthogonal right-handed coordinate system. The direction of Wind and Ulysses is defined by the longitude $\varphi$ and latitude $\theta$ relative to the source frame.

Fig. 2. The flux density time profile of a type III burst observed by Wind (top) and Ulysses (bottom) on 17 June 1996 at $196 \mathrm{kHz}$. The continuous curve above background represents the actual burst intensity profile. The lower curve (full circles), denoted $S_{\mathrm{W}}(t)$ and $S_{\mathrm{U}}(t)$, shows the same burst profile, but with the background removed. The burst is seen to occur between the onset time $t_{\text {onset }}$ and end time $t_{\text {end }}$ (see text for exact definition).

In the source reference frame defined above in Fig. 1 (right panel), we can determine the energy density $E_{i}\left(\mathrm{JHz}^{-1}\right)$ radiated by the source at frequency $f$ with flux density $S_{i}(t)$ from $t_{\text {onset }}$ to $t_{\text {end }}$ (Fig. 2), per unit solid angle in the direction $\left(\varphi_{i}, \theta_{i}\right)$ of the spacecraft at distance $d_{i}$ :

$E_{i}\left(f, \varphi_{i}, \theta_{i}\right)=\int_{t_{\text {onset }}}^{t_{\text {end }}} S_{i}\left(t, f, \varphi_{i}, \theta_{i}\right) \mathrm{d}_{i}^{2} \mathrm{~d} t$.

This can be defined as

$E_{i}\left(f, \varphi_{i}, \theta_{i}\right)=E_{\max }(f) D\left(f, \varphi_{i}, \theta_{i}\right)$

where $D\left(f, \varphi_{i}, \theta_{i}\right)$ is the radiation diagram of the event at $f$, normalized to 1 in the direction of maximum intensity, and $E_{\max }(f)$ the energy spectrum of the event taken in the direction of maximum intensity at each frequency.

From the above, it is clear that we cannot use the measurements of the energy density from a single spacecraft to derive the directivity, since the energy $E_{\max }(f)$ varies widely from one event to another. Then for measuring the radiation diagram, we consider the radio energy ratios at a frequency $f$ between Ulysses (index U) and Wind (index W) in order to eliminate $E_{\max }(f)$, which is not actually measured, such as,

$$
\begin{aligned}
R_{\mathrm{W}}(f) & =\frac{E_{\mathrm{W}}\left(f, \varphi_{\mathrm{W}}, \theta_{\mathrm{W}}\right)}{E_{\mathrm{U}}\left(f, \varphi_{\mathrm{U}}, \theta_{\mathrm{U}}\right)}=\frac{E_{\max }(f) D\left(f, \varphi_{\mathrm{W}}, \theta_{\mathrm{W}}\right)}{E_{\max }(f) D\left(f, \varphi_{\mathrm{U}}, \theta_{\mathrm{U}}\right)} \\
& =\frac{D\left(f, \varphi_{\mathrm{W}}, \theta_{\mathrm{W}}\right)}{D\left(f, \varphi_{\mathrm{U}}, \theta_{\mathrm{U}}\right)} \\
R_{\mathrm{U}}(f) & =\frac{1}{R_{\mathrm{W}}(f)}=\frac{E_{\mathrm{U}}\left(f, \varphi_{\mathrm{U}}, \theta_{\mathrm{U}}\right)}{E_{\mathrm{W}}\left(f, \varphi_{\mathrm{W}}, \theta_{\mathrm{W}}\right)}=\frac{E_{\max }(f) D\left(f, \varphi_{\mathrm{U}}, \theta_{\mathrm{U}}\right)}{E_{\max }(f) D\left(f, \varphi_{\mathrm{W}}, \theta_{\mathrm{W}}\right)} \\
& =\frac{D\left(f, \varphi_{\mathrm{U}}, \theta_{\mathrm{U}}\right)}{D\left(f, \varphi_{\mathrm{W}}, \theta_{\mathrm{W}}\right)} .
\end{aligned}
$$

Equations (4) and (5) imply that the measured energy ratios, $R_{\mathrm{W}}$ and $R_{\mathrm{U}}$, are equal to the ratio of the radiation diagram observed by the two spacecraft in two different directions $\left(\varphi_{\mathrm{W}}, \theta_{\mathrm{W}}\right)$ and $\left(\varphi_{\mathrm{U}}, \theta_{\mathrm{U}}\right)$. It is noteworthy that it is not redundant to use both $R_{\mathrm{W}}$ and $R_{\mathrm{U}}$ because they put two times more constraints on the diagram to be measured, as we shall see in the next section. 
Table 1. Characteristics of the type III radiation diagram.

\begin{tabular}{cccccc}
\hline \hline Frequency (kHz) & Data points & $\varphi_{0}(\mathrm{deg})$ & $a$ & $\Delta \varphi_{2}(\mathrm{deg})$ & $\Delta \varphi_{10}(\mathrm{deg})$ \\
$940-740$ & 2020 & $-23 \pm 1$ & $0.92 \pm 0.08$ & $96 \pm 2$ & $190 \pm 4$ \\
$540-388$ & 1900 & $-27 \pm 1$ & $0.87 \pm 0.09$ & $98 \pm 2$ & $196 \pm 4$ \\
$272-196$ & 822 & $-29 \pm 2$ & $0.78 \pm 0.11$ & $104 \pm 4$ & $212 \pm 8$ \\
$148-124$ & 344 & $-35 \pm 5$ & $0.77 \pm 0.13$ & $106 \pm 4$ & $216 \pm 14$ \\
$104-80$ & 212 & $-55 \pm 6$ & $0.50 \pm 0.20$ & $124 \pm 12$ & $352 \pm 16$ \\
\hline
\end{tabular}

\section{Results}

In this section, we derive the radiation diagram $D(f, \varphi, \theta)$ from the measurements of the radio energy ratios, $R_{\mathrm{W}}$ and $R_{\mathrm{U}}=1 / R_{\mathrm{W}}$ (Eqs. (4) and (5)), made simultaneously by Wind in the direction $\left(\varphi_{\mathrm{W}}, \theta_{\mathrm{W}}\right)$ and Ulysses in the direction $\left(\varphi_{\mathrm{U}}, \theta_{\mathrm{U}}\right)$ relative to the source frame, as illustrated in Fig. 1. For that purpose, we fit a model function with 2 fitting parameters, which will be explained and justified in Sect. 4.2, to the energy ratios $R_{\mathrm{W}}$ and $R_{\mathrm{U}}$. In the next section, in order to guide the choice of the model diagram to be fitted to observations, we highlight the complex dependency of the observables $R_{\mathrm{W}}$ and $R_{\mathrm{U}}$ upon the directions $\left(\varphi_{\mathrm{W}}, \theta_{\mathrm{W}}\right)$ and $\left(\varphi_{\mathrm{U}}, \theta_{\mathrm{U}}\right)$ of Wind and Ulysses.

\subsection{Energy ratio vs. observing direction}

To show how the energy ratios $R_{\mathrm{W}}$ and $R_{\mathrm{U}}$ and the related diagram $D$ may depend on the observing direction $(\varphi, \theta)$, Fig. 3 represents the data $R_{\mathrm{W}}$ and $R_{\mathrm{U}}$ as functions of the observing longitude $\varphi$ and latitude $\theta$. Figure 3a displays measurements of $R_{\mathrm{W}}$ and $R_{\mathrm{U}}$ at all frequencies selected between 940 and $80 \mathrm{kHz}$ as a function of $\varphi_{\mathrm{W}}$ and $\varphi_{\mathrm{U}}$ respectively, for different absolute values of $\theta_{\mathrm{W}}$ and $\theta_{\mathrm{U}}$, respectively (coded by the color bar scale). Similarly Fig. 3b shows measurements of $R_{\mathrm{W}}$ and $R_{\mathrm{U}}$ at all frequencies selected as a function of $\theta_{\mathrm{W}}$ and $\theta_{\mathrm{U}}$, respectively, for different absolute values of $\varphi_{\mathrm{W}}$ and $\varphi_{\mathrm{U}}$. To check for any dependence on direction $(\varphi, \theta)$ of the $R$ data, their averaged values in $20^{\circ}$ bins of the abscissae $\varphi$ and $\theta$ are also shown.

As can be seen from Fig. $3 \mathrm{~b}, R$ shows a slight variation with the observing latitude $\theta$ : the $\theta$ averaged values of $R$ tend to lie approximately around a flat maximum at low latitudes and decrease slightly by a factor of 6 towards higher latitudes. In contrast, Fig. 3a displays a stronger dependence by $R$ on the observing longitude $\varphi$ : the $\varphi$ averaged values of $R$ decrease from a maximum at small longitudes to a minimum at large longitudes by a factor of 25. Furthermore, the relative latitude $\theta$ appears to be uniformly distributed about the $\varphi$ variation trend of $R$ : this means that the variation of $R$ with $\varphi$ is independent of $\theta$.

\subsection{Radiation diagram vs. observing longitude}

Figure 3 a suggests that the energy ratios $R$ and radiation diagram $D$, to a first approximation, are only functions of the observing longitude $\varphi$ and frequency $f$. From Eqs. (4) and (5), and omitting $f$ for brevity, the $R_{\mathrm{W}}$ and $R_{\mathrm{U}}$ data can be expressed by the model functions:

$$
\begin{aligned}
& \mathfrak{R}_{\mathrm{W}}=\frac{D\left(\varphi_{\mathrm{W}}\right)}{D\left(\varphi_{\mathrm{U}}\right)}, \\
& \mathfrak{R}_{\mathrm{U}}=\frac{D\left(\varphi_{\mathrm{U}}\right)}{D\left(\varphi_{\mathrm{W}}\right)} .
\end{aligned}
$$

For the radiation diagram $D(\varphi)$ itself, we take an empirical model function of the form

$D(\varphi)=10^{a\left[\cos \left(\varphi-\varphi_{0}\right)-1\right]}$, where $\varphi$ is the observing longitude $\left(\varphi_{\mathrm{W}}, \varphi_{\mathrm{U}}\right), \varphi_{0}$ the longitude of the maximum intensity (normalized to 1 ), and $a$ the amplitude of the cosine diagram. Here, $a$ defines the full angular width $\Delta \varphi_{n}$ at the maximum $/ n$ level of the diagram such that

$$
\begin{aligned}
& \frac{1}{n}=10^{a\left[\cos \left(\Delta \varphi_{n} / 2\right)-1\right]} \\
& \Delta \varphi_{n}=2 \arccos \left(1-\log _{10} n / a\right)
\end{aligned}
$$

The choice of the model diagram in Eq. (8) is simply based on the shape with a single beam exhibited by the observed $R_{\mathrm{W}}$ and $R_{\mathrm{U}}$ represented in Fig. 3. It is worth noticing that the observed diagram is nothing compared to the primary diagram at the source because of the ubiquitous propagation effects in the medium, which are expected to strongly distort and smooth out any sophisticated primary diagram that exists.

We then fit the model functions $\mathfrak{R}_{\mathrm{W}}$ and $\mathfrak{R}_{\mathrm{U}}$, with the two fitting parameters $a$ and $\varphi_{0}$, to the measured $R_{\mathrm{W}}$ and $R_{\mathrm{U}}$ data (Eqs. (4) and (5) and Fig. 3a), by the Levenberg-Marquardt nonlinear least-squares method (Press et al. 1992). The results of the diagram fitting are shown in Fig. 4 for five groups of two frequencies: (a) 940-740, (b) 540-388, (c) 272-196, (d) 148124 , and (e) $104-80 \mathrm{kHz}$. The diagram $D(\varphi)$ (Eq. (8)) obtained with the fitted values of $a$ and $\varphi_{0}$ is plotted as $\log _{10} D(\varphi)$ vs. $\varphi$. These values of $a$ and $\varphi_{0}$ are also given in each panel of Fig. 4 and in Table 1. Since we have no direct measurements of the diagram itself, which is obtained by fitting to the actually measured data $R_{\mathrm{W}}$ and $R_{\mathrm{U}}$, we represent the diagram data by $D_{\mathrm{W}}\left(\varphi_{\mathrm{W}}\right)=R_{\mathrm{W}} \times D\left(\varphi_{\mathrm{U}}\right)$ and $D_{\mathrm{U}}\left(\varphi_{\mathrm{U}}\right)=R_{\mathrm{U}} \times D\left(\varphi_{\mathrm{W}}\right)$. For illustration, these data are averaged in $20^{\circ}$ bins of $\varphi$ and plotted as a function of $\varphi$ in Fig. 4. As expected, the fitted curve of the diagram can be seen to clearly pass through the averaged diagram data points to within the scatter bars.

In Fig. 4, the derived value of the amplitude $a$ of the cosine diagram gives the full angular width $\Delta \varphi_{n}$ of the diagram at maximum $/ n$ level (see Eq. (10)). The derived $\varphi_{0}$ indicates an eastward tilt of the radiation diagram from the local magnetic field direction at the source. As the frequency decreases, the eastward shift $\varphi_{0}$ increases and the amplitude $a$ decreases (consequently the diagram width $\Delta \varphi_{n}$ increases). Table 1 summarizes the results of the diagram fitting assuming the emission source at the fundamental $(F)$, together with the diagram widths $\Delta \varphi_{2}\left(\right.$ at maximum/2) and $\Delta \varphi_{10}$ (at maximum/10) as computed from Eq. (10). In the table, the fitting parameters $\varphi_{0}$ and $a$ are given with their numerical uncertainties for the five groups of two frequencies. The present diagram observations essentially confirm previous results of Hoang et al. (1997) on the angular width and eastward shift of the type III emission diagram, but provide more accurate diagrams thanks to systematic comparisons of Wind and Ulysses radio burst data at the same frequencies and in all directions. Further consideration of these diagram characteristics will be given in Sect. 5 . 
(a)

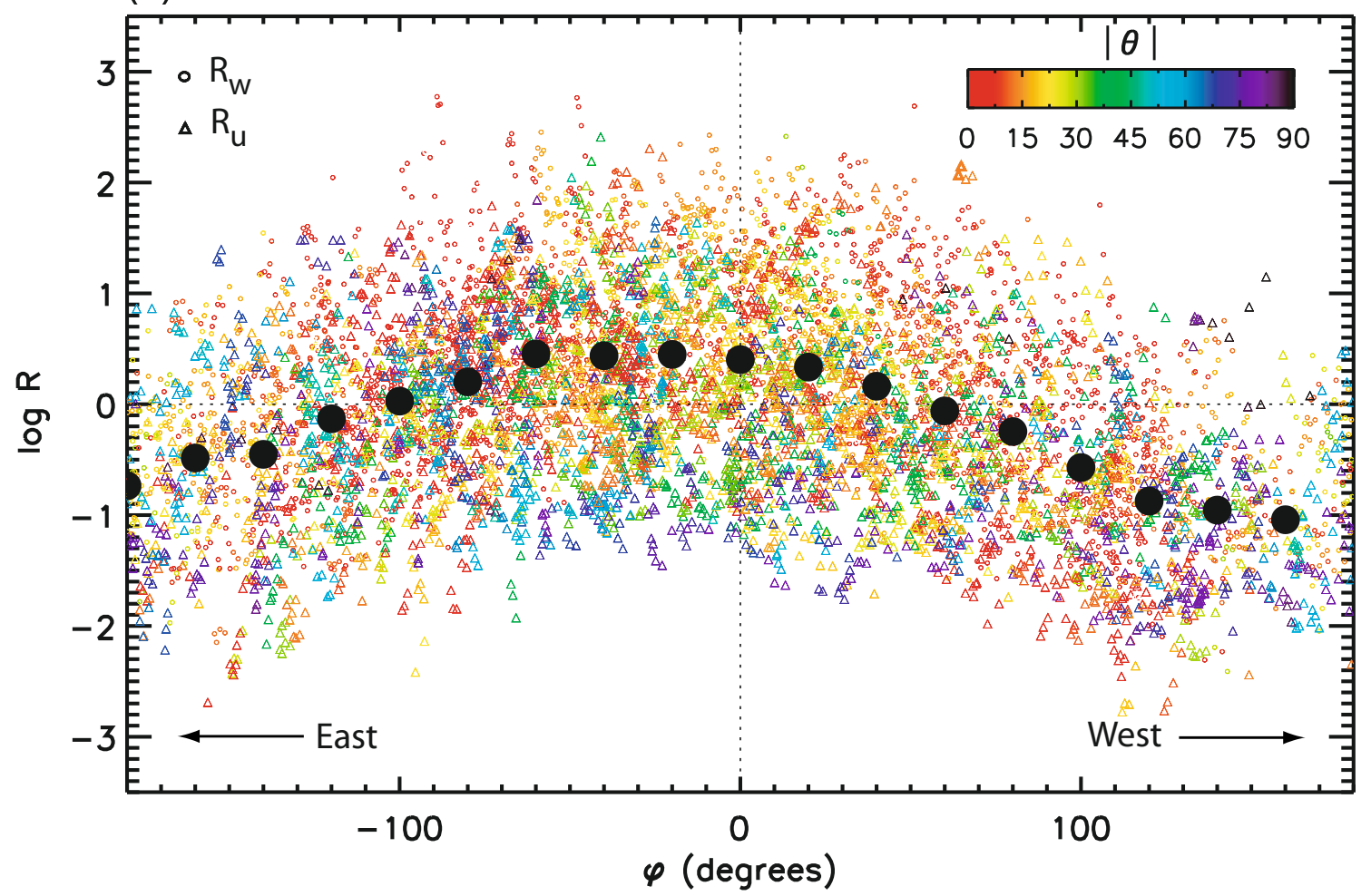

(b)

$1995-2005$

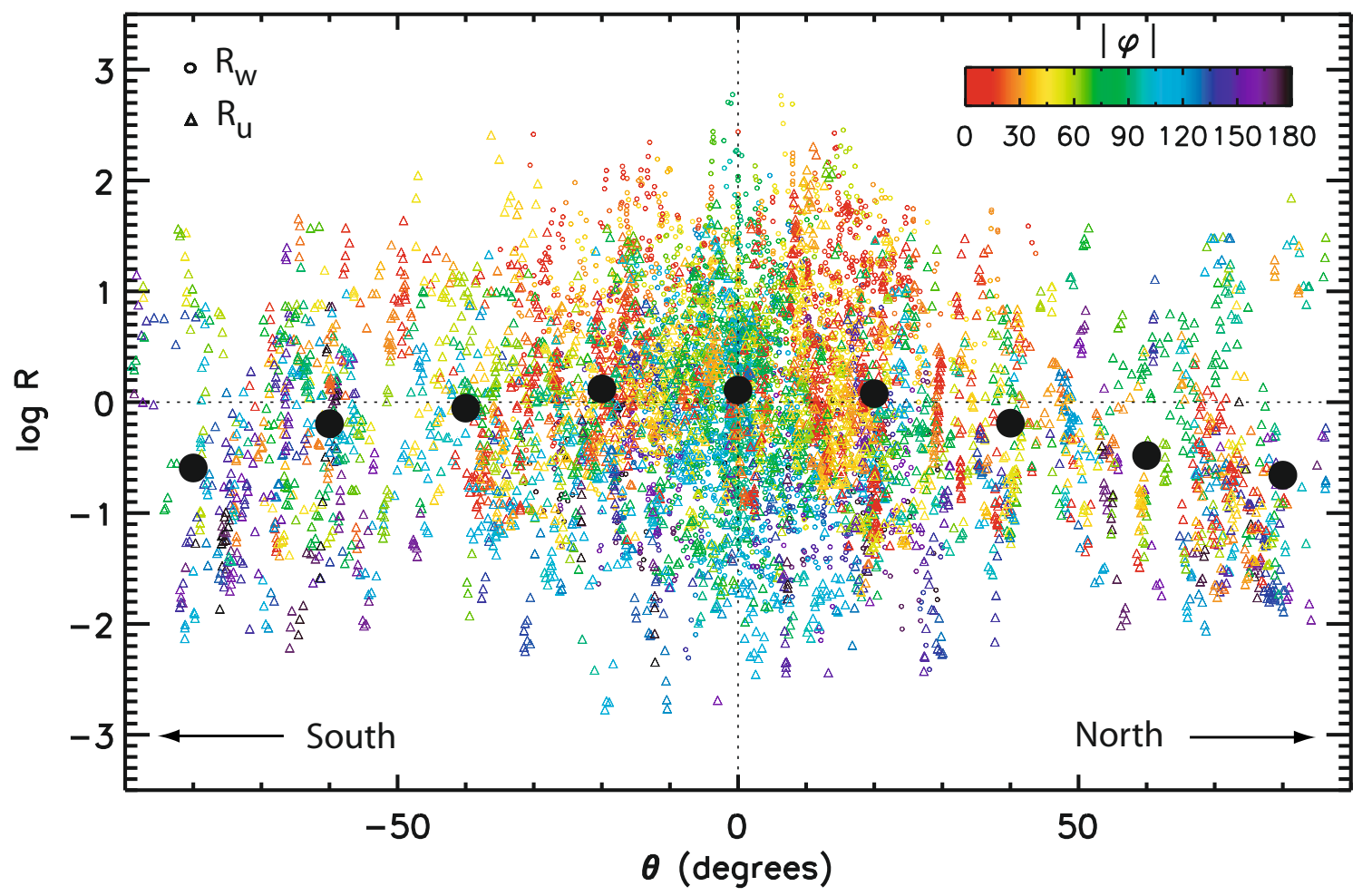

Fig. 3. (a) Type III burst energy ratios $R$ measured from Wind (circles) and Ulysses (triangles), at all selected frequencies and for all relative latitudes $\theta$ of the spacecraft, $\theta_{\mathrm{W}}$ and $\theta_{\mathrm{U}}$, as a function of the longitudes $\varphi$ of Wind and Ulysses, $\varphi_{\mathrm{W}}$ and $\varphi_{\mathrm{U}}$, relative to the source (in coordinates $x^{\prime} y^{\prime} z^{\prime}$, Fig. 1). Different absolute values of latitude $\theta$ are indicated by a color bar. Averaged values of $R$ in $20^{\circ}$ bins of longitude $\varphi$ are plotted as black full circles. (b) Energy ratios $R$ measured from Wind (circles) and Ulysses (triangles), at all selected frequencies and for all relative longitudes $\varphi$ of the spacecraft, $\varphi_{\mathrm{W}}$ and $\varphi_{\mathrm{U}}$, as a function of the latitudes $\theta$ of Wind and Ulysses, $\theta_{\mathrm{W}}$ and $\theta_{\mathrm{U}}$, relative to the source. Different absolute values of $\varphi$ indicated by the color bar. Averaged values of $R$ in $20^{\circ}$ bins of $\theta$ plotted as black full circles. 
$1995-2005$
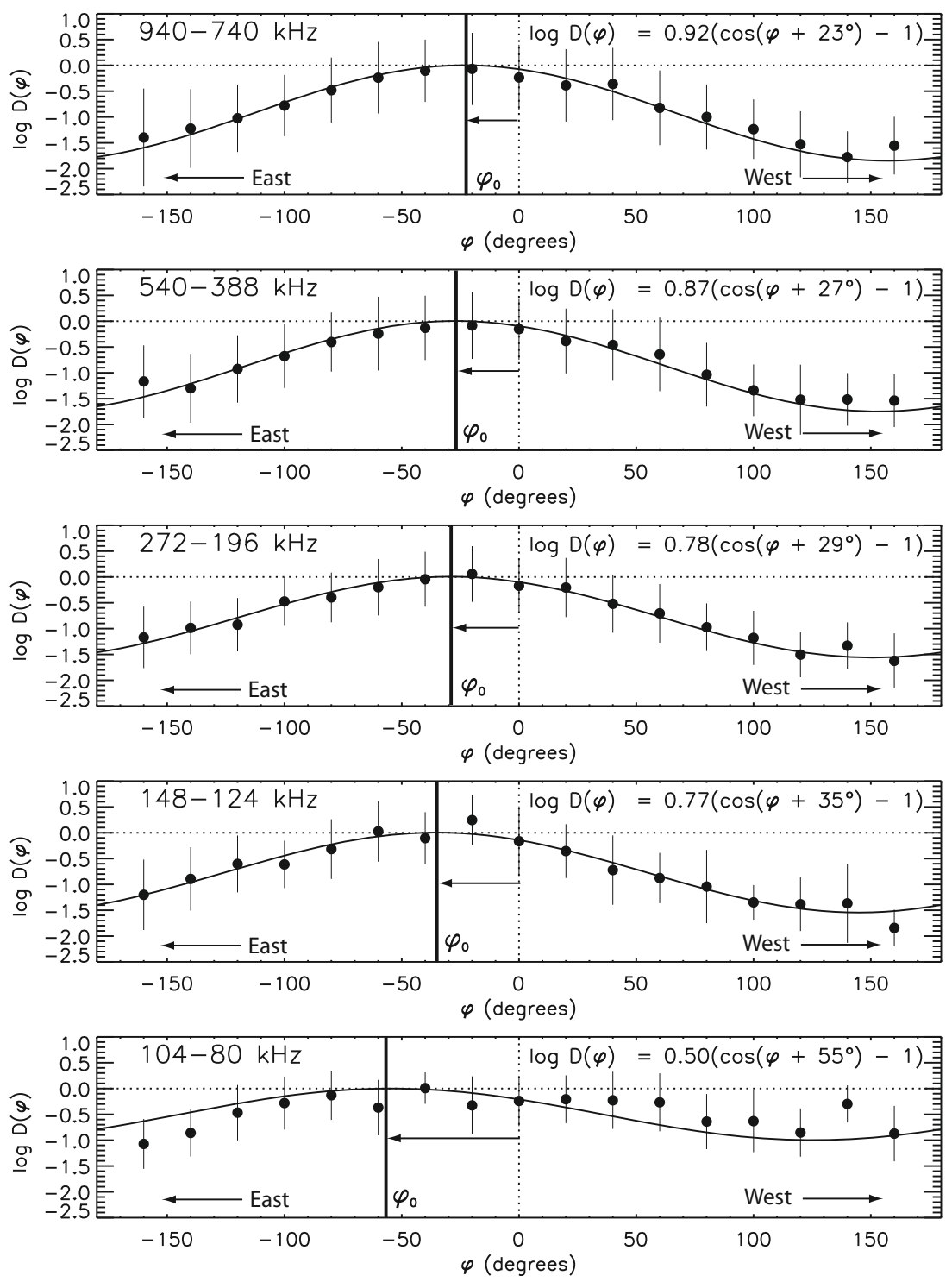

Fig. 4. The diagram in longitude $\varphi$ relative to the source, with the fitted values of the amplitude $a$ and shift $\varphi_{0}$, is plotted as a continuous line as $\log _{10} D(\varphi)$ vs. $\varphi$, in different frequency ranges. The diagram data (see definition in the text) are averaged in $20^{\circ}$ bins of $\varphi$ and plotted (black circles with scatter bars) vs. $\varphi$. As the frequency decreases, the shift $\varphi_{0}$ to east of the local magnetic field direction increases and the amplitude $a$ of the cosine diagram decreases.

\subsection{Effect of the observing latitude on the directivity}

In this section, we examine the latitude dependence of the radiation diagram in more detail. From the above section and Eqs. (4) and (5), we may express the energy ratios differently as measured between Wind (W) and Ulysses (U) (at $f$ ) by

$R_{\mathrm{W}}=\frac{D\left(\varphi_{\mathrm{W}}\right)}{D\left(\varphi_{\mathrm{U}}\right)} \frac{D\left(\theta_{\mathrm{W}}\right)}{D\left(\theta_{\mathrm{U}}\right)}$,

$R_{\mathrm{U}}=\frac{D\left(\varphi_{\mathrm{U}}\right)}{D\left(\varphi_{\mathrm{W}}\right)} \frac{D\left(\theta_{\mathrm{U}}\right)}{D\left(\theta_{\mathrm{W}}\right)}$,

where the model diagram function $D(\theta)$ has the same form as $D(\varphi)$ of Eq. (8). Then by removing the longitude $\varphi$ dependence from the diagram, we can obtain the energy ratios that are functions of the latitude $\theta$ alone as

$R_{\mathrm{W}}^{\theta}=R_{\mathrm{W}} \frac{D\left(\varphi_{\mathrm{U}}\right)}{D\left(\varphi_{\mathrm{W}}\right)}=\frac{D\left(\theta_{\mathrm{W}}\right)}{D\left(\theta_{\mathrm{U}}\right)}$,

$R_{\mathrm{U}}^{\theta}=\frac{1}{R_{\mathrm{W}}^{\theta}}=\frac{D\left(\theta_{\mathrm{U}}\right)}{D\left(\theta_{\mathrm{W}}\right)}$
The $R_{\mathrm{W}}^{\theta}$ and $R_{\mathrm{U}}^{\theta}$ ratios can then be expressed by the empirical model functions, which depend on the latitude $\theta$ alone:

$\mathfrak{R}_{\mathrm{W}}^{\theta}=\frac{D\left(\theta_{\mathrm{W}}\right)}{D\left(\theta_{\mathrm{U}}\right)}$

$\mathfrak{R}_{\mathrm{U}}^{\theta}=\frac{D\left(\theta_{\mathrm{U}}\right)}{D\left(\theta_{\mathrm{W}}\right)}$

with

$D(\theta)=10^{b\left[\cos \left(\theta-\theta_{0}\right)-1\right]}$,

where $\theta_{0}$ is the latitude $\theta$ of the maximum intensity (normalized to 1 ), and $b$ the amplitude of the cosine diagram.

We then fit the model functions $\mathfrak{R}_{\mathrm{W}}^{\theta}$ and $\mathfrak{R}_{\mathrm{U}}^{\theta}$, with the fitting parameters $b$ and $\theta_{0}$, to the measured $R_{\mathrm{W}}^{\theta}$ and $R_{\mathrm{U}}^{\theta}-$ Eqs. (13) and (14). The results of the diagram fitting are shown in Fig. 5 for the five groups of two frequencies: (a) 940-740, (b) 540-388, (c) 272-196, (d) 148-124, and (e) 104-80 kHz. The diagram $D(\theta)$ (Eq. (17)) obtained with the fitted values of $b$ and $\theta_{0}$ is plotted in continuous line as $\log _{10} D(\theta)$ vs. $\theta$. These values of $b$ and $\theta_{0}$ are also displayed in each panel of Fig. 5. Since we 
$1995-2005$
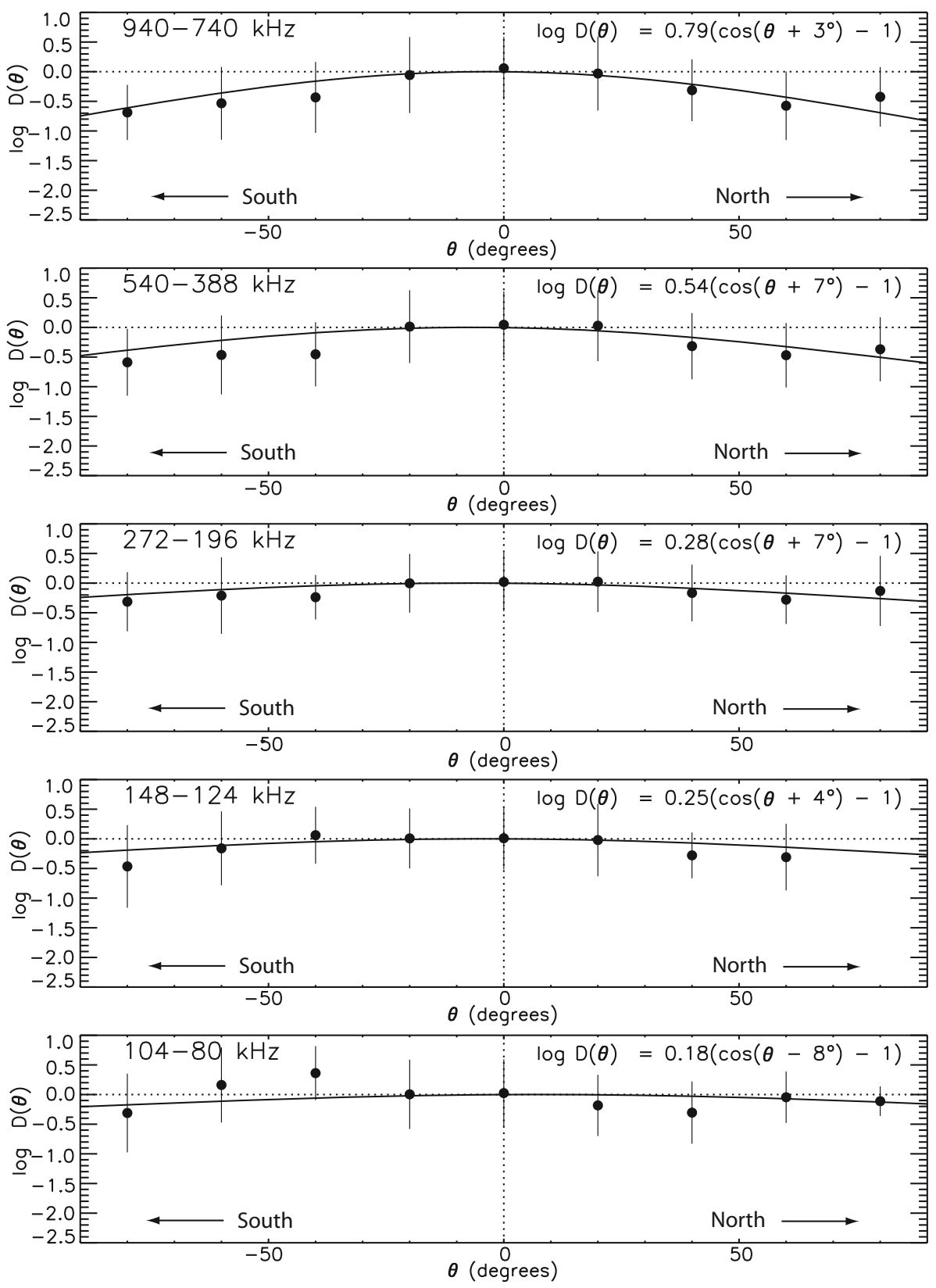

Fig. 5. The diagram in latitude $\theta$ relative to the source, with the fitted values of the amplitude and shift, is plotted as a continuous line as $\log _{10} D(\theta)$ vs. $\theta$, in different frequency ranges. The diagram data (see definition in the text) are averaged in $20^{\circ}$ bins of $\theta$ and plotted (black circles with scatter bars) vs. $\theta$. No significant tilt is seen in the diagram. A weak directivity is observed equatorward at highest frequencies. At low frequency, the diagram is nearly isotropic.

have no direct measurements of the diagram that is obtained by least-squares fitting to the actually measured data $R_{\mathrm{W}}^{\theta}$ and $R_{\mathrm{U}}^{\theta}$, we represent the diagram data by $D_{\mathrm{W}}\left(\theta_{\mathrm{W}}\right)=R_{\mathrm{W}}^{\theta} \times D\left(\theta_{\mathrm{U}}\right)$ and $D_{\mathrm{U}}\left(\theta_{\mathrm{U}}\right)=R_{\mathrm{U}}^{\theta} \times D\left(\theta_{\mathrm{W}}\right)$. For illustration, the latter data are averaged in $20^{\circ}$ bins of $\theta$ and plotted as a function of $\theta$ in Fig. 5. The fitted curve of the diagram can be seen, as expected, to pass through the diagram's averaged data points to within the scatter bars. From the figure, there is no significant tilt $\theta_{0}$ of the diagram maximum from $0^{\circ}$ latitude, in contrast to the longitude $\varphi$ case. The amplitude $b$ of the cosine diagram $D(\theta)$ is also much smaller than the amplitude $a$ of the diagram $D(\varphi)$ and decreases rapidly towards lower frequencies. This will be discussed further in Sect. 5.3.

\section{Discussion}

We now address different effects that may affect the type III radiation diagram we have found above.

\subsection{Eastward shift of the radiation diagram}

From Fig. 4 we find that the radiation diagram axis shifts significantly to east from the local magnetic field direction, in agreement with previous observations of Poquérusse et al. (1996) and Hoang et al. (1997). This eastward shift of the diagram axis out of the spiral magnetic field lines traveled by the type III electrons is not consistent with what should be expected from the plasma emission mechanism of the type III bursts that are emitted in that magnetic field direction. Following Poquérusse et al. (1996) and referring to Fig. 6, we can interpret the eastward shift of the diagram axis as follows. The type III electron streams travel outward along open-field lines from the associated flare sites in active regions through complex coronal structures of closed-field lines. Along these open-field lines, the solar wind escapes faster than in the surrounding areas. At some distance farther out, when the spiral open-field lines start to deviate by a few degrees from the radial direction, the faster wind along the type III electron trajectories catches up with the ambient slower wind, and thus 


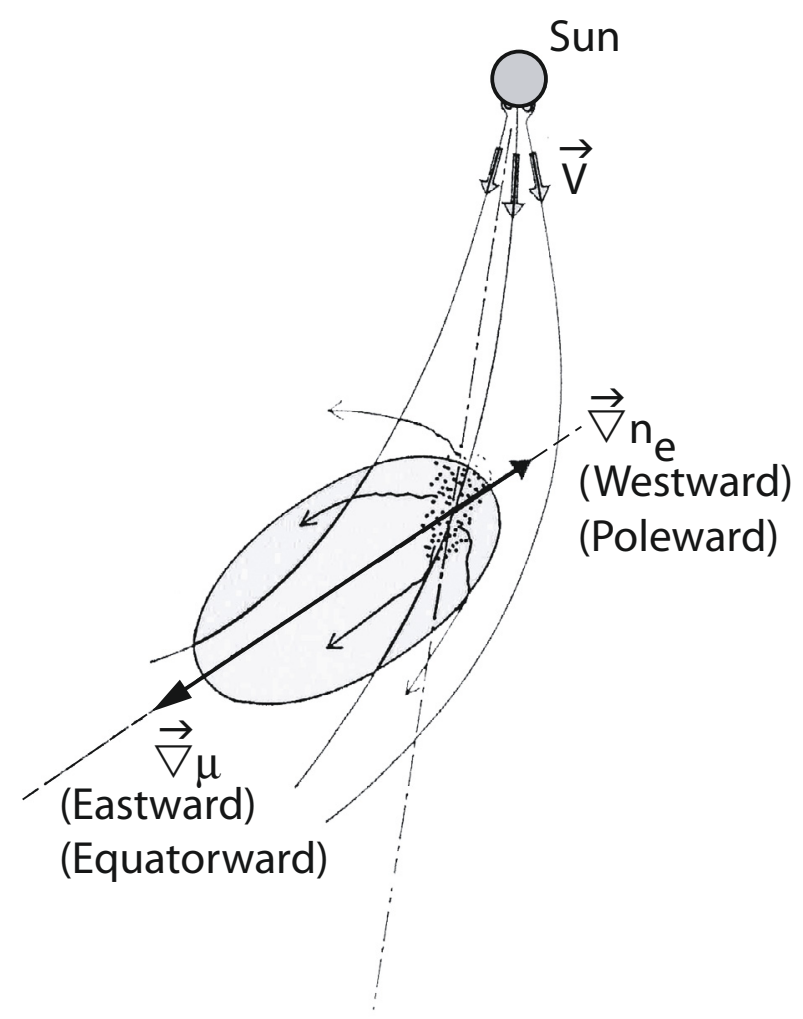

Fig. 6. Schematic interpretation of the eastward (equatorward) shift of the type III emission diagram in longitude (latitude) relative to the local open-field direction at the source. The type III electrons travel outward along spiral open-field lines from the associated flare sites in active regions. Along these paths, the solar wind escapes faster than in the surrounding areas filled with structures of closed-field lines. At some distance farther out, the faster wind catches up with the ambient slower wind and produces a density compression that leads to a transverse density gradient $\nabla n_{\mathrm{e}}$ directed westward (poleward) of the open-field direction. This in turn results in a refractive index gradient $\nabla \mu=-\nabla n_{\mathrm{e}}$, oriented eastward (equatorward) in the opposite sense of the density gradient. Radiation is thus bent in the eastward (equatorward) direction as observed.

produces a density compression that leads to a transverse density gradient $\nabla n_{\mathrm{e}}$ directed west of the spiral field direction, like in the well-known corotating interaction regions (CIRs). This in turn results in a refractive index gradient $\nabla \mu=-\nabla n_{\mathrm{e}}$, oriented eastward in the opposite sense of the density gradient, and so bends radiation in the eastward direction as observed.

It is well known that, in the CIR density compression regions observed in the solar wind, westward density gradients actually exists (e.g., Schwenn \& Marsch 1990, Fig. 3.10; Lacombe et al. 2000, Fig. 2). On the other hand, that the eastward shift of the diagram increases with decreasing frequency (Fig. 4 and Table 1), i.e., at larger heliocentric distance, implies that the density gradient (or density compression) along the CIR-like path traveled by the type III electrons is presumably stronger farther away from the sun. This seems to be suggested by three-dimensional magnetohydrodynamic models of solar wind large-scale structures (Pizzo 1994).

\subsection{Directivity and propagation effects}

As summarized in Table 1, the angular width of the type III radiation diagram increases with decreasing observing frequencies.
This is presumably due to propagation effects at work in the IPM: refraction by regular density gradient and scattering by random density fluctuations. Computer simulations of these propagation effects have been performed for several decades, among other emission features, on the directivity at hectometer and kilometer wavelengths (e.g., Steinberg 1972; Thejappa et al. 2007). Using a simple model of isotropic point source and different density relative fluctuations with Gaussian distribution (Steinberg 1972) or power-law distribution (Thejappa et al. 2007), by computer Monte Carlo simulations these authors could qualitatively reproduce the anisotropic shape and large width of the apparent directivity diagram of the type III at low frequency.

There can be no doubt therefore that, on the grand average, propagation effects are likely to strongly affect the primary diagram of the type III source. Detailed propagation computations, taking additional parameters into account such as the source angular size and beaming, are necessary before attempting to deduce any information on the radiation diagram of the primary source (e.g., Zheleznyakov \& Zaitsev 1970).

Furthermore, since energy loss due to electron collisions can be neglected in the IPM, we might expect propagation effects to be negligible in the radiation transfer at low frequency, while they do strongly distort the radiation diagram because of angular scattering undergone by radio waves. We can thus reasonably assume that the total energy radiated in space by the emission source is not much affected by propagation. This consideration is worthy of note when estimating the efficiency of the mode conversion in the type III emission mechanism.

\subsection{Directivity and the observing relative latitude}

As can be seen from Fig. 5, the amplitude of the latitude diagram is found to be much smaller than that of the longitude diagram illustrated in Fig. 4: this is consistent with the dominant effect of the longitude in the type III directivity diagram. On the other hand, this amplitude decreases rapidly from higher frequencies to lower frequencies, the diagram in latitude becomes isotropic at low frequency. At highest frequencies, the latitude diagram is slightly anisotropic, decreasing from maximum around $0^{\circ}$ latitude to about maximum $/ 6$ at high latitude. This latitude behavior of the emission, which increases towards low latitude (equatorward), was also observed by Hoang et al. (1997). As observed, this latitudinal directivity appears to be contrary to that expected from a plasma density distribution more or less concentrated in a dense and narrow solar equatorial band as actually observed at high latitude (see Issautier et al. 1997, and references therein). Indeed, such a plasma density distribution presents a latitudinal component of the density gradient directed towards low latitude and consequently should bend radiation in the opposite sense, i.e., towards high latitude (poleward).

To explain the equatorward directivity, as in the case of the eastward shift of the longitude diagram discussed in Sect. 5.1 and shown in Fig. 6, we speculate on a poleward component of the local density gradient $\nabla n_{\mathrm{e}}$ along the type III electron openfield tube. As a result, this will produce a refractive index gradient $\nabla \mu=-\nabla n_{\mathrm{e}}$ and bend radiation equatorwards.

\subsection{Directivity and the type III radiation mode}

From stereoscopic observations of type III bursts (see Dulk 2000, and references therein), it has been suggested that type III bursts radiate at both the fundamental $(\mathrm{F})$ and harmonic $(\mathrm{H})$, but with different directivities: at small (longitude) angles from 
the spiral type III electron trajectory, the radiation at a given frequency is initially at $\mathrm{F}$ and followed later on by $\mathrm{H}$; at large angles, only $\mathrm{H}$ is observed. These $\mathrm{F}$ and $\mathrm{H}$ directivities have been established by Monte Carlo simulation of radio wave propagation in the IPM (Thejappa et al. 2007).

As a consequence, the directivity we measure at the same frequency $f$ might consist of both the fundamental $\mathrm{F}$ and harmonic $\mathrm{H}$ radiation near the diagram axis; and way off the diagram axis, it might consist of $\mathrm{H}$ radiation alone. To check for that effect, we performed the data analysis by restricting the observing longitude angles to a range between $-80^{\circ}$ and $60^{\circ}$ around the diagram axis. The results found indicate higher values of the diagram amplitude $a$ and lower ones for the angular width $\Delta \varphi$ than in the case of a widespread longitude range, and thus point to the $\mathrm{F}$ narrower diagram on top of the $\mathrm{H}$ wider diagram.

\subsection{Variation of the directivity with the solar cycle}

To check for a possible variation of the radiation diagram with the solar cycle, we have divided the whole period of observation from 1995 to 2005 into two subperiods of equal duration spanning the 1996 minimum and 2001 maximum separately. We then performed the diagram data analysis on each of these subperiods. The comparison of the results found for the two subperiods and the whole period show no significant difference between the diagrams measured during the minimum and maximum phases of the solar cycle.

\section{Conclusion}

Using radio data recorded simultaneously by the Wind and Ulysses spacecraft from 1995 to 2005 at the same frequencies between 940 and $80 \mathrm{kHz}$, we have derived for the first time the average radiation diagram of the solar hectometric and kilometric type III bursts in two dimensions (longitude and latitude relative to the source). No variation with the solar cycle has been detected in the diagrams derived separately from the minimum and maximum activity phases.

The full angular width of the diagram in longitude at the maximum/10 level is about $190^{\circ}$ at $940-740 \mathrm{kHz}$ and increases to nearly $360^{\circ}$ at $104-80 \mathrm{kHz}$. The axis of the diagram is found to shift eastward from the local magnetic field direction at the source by an angle increasing from $23^{\circ}$ at $940-740 \mathrm{kHz}$ to $55^{\circ}$ at $104-80 \mathrm{kHz}$. This eastward shift is interpreted to be the result of refraction by a westward density gradient due to a local density compression region along the open magnetic field lines above the associated active regions.

The diagram in latitude, obtained uniquely thanks to the outof-ecliptic exploration of Ulysses, shows no poleward directivity that should be expected from the interplanetary plasma density distribution, more or less spherical, that exists at latitudes above the ecliptic plane. On the contrary, at the highest frequencies this diagram is observed to decrease slightly towards higher latitudes; at lower frequencies, it becomes nearly isotropic with latitudes. The absence of a poleward directivity in latitude is possibly due to a local poleward density gradient that results in a local equatorward refractive index gradient bending radiation towards low latitudes.

Our study has confirmed previous findings of Hoang et al. (1997) from the Ulysses/URAP radio receiver and ARTEMIS ground-based radio spectrograph. Moreover, it has provided more accurate measurements of the average radiation diagram of the type III bursts and new insights into the propagation of radio waves and solar particles along open magnetic field lines above active regions and in corotating interaction regions. In this respect, our results implying transverse density compression bring new constraints to hydrodynamic models dealing with solar wind structures (see Forsyth \& Gosling 2001, and references therein). Precise knowledge of this diagram obtained with our study will be valuable for studying both the emission mechanism and the propagation in interplanetary space of the type III bursts. The data analysis tool developed for the present study can be easily applied to the STEREO duo-spacecraft mission to investigate solar radio emissions.

Acknowledgements. The Wind/WAVES investigation is a collaboration of the Observatoire de Paris, NASA/GSFC, and the University of Minnesota. The Ulysses/URAP investigation is a joint project of NASA/GSFC, the Observatoire de Paris, the University of Minnesota, and the CETP, Velizy, France. The French contribution to these projects is supported by the CNES and CNRS. Part of the data used in this study comes from the Centre des Données de la Physique des Plasmas (CDPP) in Toulouse, France. We are grateful to Catherine Lacombe and Carine Briand for careful reading of the manuscript and helpful comments. S.H. thanks Michel Poquérusse for interesting discussions on the type III emission directivity.

\section{References}

Bougeret, J.-L., Kaiser, M. L., Kellogg, P. J., et al. 1995, Space Sci. Rev., 71, 231

Caroubalos, C., \& Steinberg, J. L. 1974, A\&A, 32, 245

Caroubalos, C., Poquérusse, M., \& Steinberg, J. L. 1974, A\&A, 32, 255

Dulk, G. A. 2000, in Radio Astronomy at Long Wavelengths, ed. R. G. Stone, K. W. Weiler, M. L. Goldstein, \& J.-L. Bougeret, 115

Dulk, G. A., Steinberg, J. L., Lecacheux, A., Hoang, S., \& MacDowall, R. J. 1985, A\&A, 150, L28

Fainberg, J., Hoang, S., \& Manning, R. 1985, A\&A, 153, 145

Forsyth, R. J., \& Gosling, J. T. 2001, Corotating and transient structures in the heliosphere (The heliosphere near solar minimum. The Ulysses perspective, ed. A. Balogh, R. G. Marsden and E. J. Smith, Springer-Praxis Books in Astrophysics and Astronomy (London: Springer), 107

Hoang, S., Poquérusse, M., \& Bougeret, J.-L. 1997, Sol. Phys., 172, 307

Issautier, K., Meyer-Vernet, N., Moncuquet, M., \& Hoang, S. 1997, Sol. Phys., 172,335

Issautier, K., Meyer-Vernet, N., Moncuquet, M., \& Hoang, S. 1998, J. Geophys. Res., 103, 1969

Lacombe, C., Salem, C., Mangeney, A., et al. 2000, Ann. Geophys., 18, 852

Leblanc, Y., Dulk, G. A., \& Bougeret, J.-L. 1998, Sol. Phys., 183, 165

Lecacheux, A., Steinberg, J.-L., Hoang, S., \& Dulk, G. A. 1989, A\&A, 217, 237 MacDowall, R. J. 1982, Master's Thesis, University of Maryland

Manning, R., \& Fainberg, J. 1980, Space Sci. Instrum., 5, 161

McComas, D. J., Bame, S. J., Feldman, W. C., Gosling, J. T., \& Phillips, J. L. 1992, Geophys. Res. Lett., 19, 1291

Ogilvie, K. W., Chornay, D. J., Fritzenreiter, R. J., et al. 1995, Space Sci. Rev., 71,55

Pizzo, V. J. 1994, J. Geophys. Res., 99, 4185

Poquérusse, M. 1984, Ph.D. Thesis, University Paris 7

Poquérusse, M., Hoang, S., Bougeret, J.-L., \& Moncuquet, M. 1996, in Amer. Inst. Phys. Conf. Ser. 382, ed. D. Winterhalter, J. T. Gosling, S. R. Habbal, W. S. Kurth, \& M. Neugebauer, 62

Press, W. H., Teukolsky, S. A., Vetterling, A. W. T., \& Flannery, B. P. 1992, Numerical Recipes in C: The Art of Scientific Computing (Cambridge University Press)

Saito, K., Poland, A. I., \& Munro, R. H. 1977, Sol. Phys., 55, 121

Schwenn, R., \& Marsch, E. 1990, Physics of the Inner Heliosphere I. Large-Scale Phenomena (Physics of the Inner Heliosphere I)

Steinberg, J.-L. 1972, A\&A, 18, 382

Stone, R. G., Bougeret, J. L., Caldwell, J., et al. 1992, A\&AS, 92, 291

Thejappa, G., MacDowall, R. J., \& Kaiser, M. L. 2007, ApJ, 671, 894

Zheleznyakov, V. V., \& Zaitsev, V. V. 1970, Sov. Astron., 14, 250 